\section{Thalidomide and its analogues: comparative clinical efficacy and safety, and cost-effectiveness}

\author{
Talidomida e seus análogos: eficácia e segurança \\ clínica comparativa e custo-efetividade
}

Talidomida y sus análogos: eficacia y seguridad
clínica y coste-efectividad
Francisco J. R. Paumgartten 1

unnoticed for nearly three decades ${ }^{1}$. Appearance of "avoidable cases" of thalidomide embryopathy in Brazil prompted health authorities to issue a regulation (Directive n. 354/MS/SVS, 1997) that prohibited thalidomide prescription to women of childbearing age except in very special circumstances and under strictly controlled conditions. In 2003, the Brazilian Congress passed a law (Law n. 10,651, April 2003) that forbade the sale and/or dispensing of thalidomide in commercial pharmacies and also stated that it should be distributed to public health units/hospitals and dispensed to patients through programs approved by the Brazilian Ministry of Health. Notwithstanding the foregoing stringent regulations, at least five more cases of thalidomide embryopathy were diagnosed in babies born between 2005 and $2010^{1}$. The recurrence of birth defects associated with misuse during pregnancy, and concerns about the uncontrolled use of off label indications led the Brazilian National Agency for Sanitary Surveillance (ANVISA) to issue a set of new rules (RDC n. 11, 2011) controlling the dispensing and prescription of thalidomide. Currently, thalidomide holds a unique position in the Brazilian pharmaceutical care and regulation systems. It is the only medicine regulated by a specific Federal law that prohibits its sale and dispensing in commercial pharmacies. Moreover thalidomide is manufactured exclusively 
by a state government owned industry (Fundação Ezequiel Dias do Estado de Minas Gerais FUNED-MG) at very low production costs, and is distributed at no cost to patients through special programs approved by the Brazilian Ministry of Health.

Thalidomide first entered the US pharmaceutical market in 1998 when Celgene Co. received US Food and Drug Administration (FDA) clearance to sell Thalidomid for manifestations of ENL, a morbid condition almost inexistent in the country. The FDA decision to approve thalidomide for an unlikely use in the US was an apparent reaction to an uncontrolled use of thalidomide entering the country illegally for a variety of new therapeutic indications. In 1999, Thalidomid received "orphan drug" designation for multiple myeloma and Crohn's disease and, in 2006, FDA approved thalidomide for relapsed and refractory multiple myeloma. The European Medicines Agency (EMA) also designated Thalidomide-Celgene (in indications for multiple myeloma) an "orphan medicine" (November 2001) because of the low prevalence of the disease in the EU (1.2 per 10,000 people). In April 2008, ThalidomideCelgene received a marketing authorization valid throughout the EU.

The emergence of new clinical indications for thalidomide encouraged the quest for analogues that share its therapeutic properties but not its teratogenic effects. So far two compounds structurally related to thalidomide (developed by Celgene Co.) have entered the pharmaceutical market.

Lenalidomide was the first thalidomide-like drug that received a clearance for sales in the US (for myelodisplastic syndrome and multiple myeloma in 2005 and 2006, respectively), Europe (2013) and in other countries. Although FDA and EMA gave lenalidomide an "orphan drug" status (for myelodisplastic syndrome and multiple myeloma), it proved to be a very profitable product (Revlimid was a Celgene's blockbuster with US $\$ 3.77$ billion in 2012 sales) 2. In 2013, a second thalidomide analogue, pomalidomide (US brand name Pomalyst), was approved for use in advanced multiple myeloma by the FDA and EMA.

Contrasting to US and EU regulatory agencies, the Brazilian agency ANVISA rejected (in 2010) a Zodiac Co. (company licensed by Celgene to sell Revlimid in Brazil) application for lenalidomide use in multiple myeloma and myelodisplastic syndrome. In 2012, a request for reconsideration filed by Zodiac Co. was denied by the agency.

Owing to Brazil's tragic experience with thalidomide, putting into the market thalidomidelike new drugs is a particularly hard decision for health authorities. It has to stand on the best evidence that the new product is clinically superior (in terms of efficacy and/or safety) to medicines already on the market, and also on unbiased cost-effectiveness analyses.

As far as thalidomide analogues are concerned, three crucial questions need to be addressed for approving a new drug application: (1) What are the risks for the embryo and fetus?; (2) Is the new drug more effective and/or safer than thalidomide? and; (3) Is the thalidomidelike drug cost effective compared to existing therapies?

A comparative study of the developmental toxicity of thalidomide and lenalidomide in rabbits found that the former drug caused structural anomalies (limb defects and others) at doses that were nontoxic to the mother whereas the latter did not increase the incidence of malformations and induced other embryotoxic effects only at maternally toxic doses. These results were initially misinterpreted as an indication that lenalidomide would be "less teratogenic" than thalidomide. A further comparative study, however, showed that lenalidomide and thalidomide caused the same type of limb reduction defects in non-human primates as those observed in humans exposed in utero to thalidomide.

After remaining a mystery for half a century the mode by which thalidomide impairs embryo development was recently deciphered. In 2010, Takumi Ito and colleagues noted that thalidomide initiated its detrimental effects on the embryo by binding to a protein (cereblon) and inhibiting the associated ubiquitin ligase activity ${ }^{3}$. A set of recently published experimental and clinical studies showed that binding of thalidomide or lenalidomide to the same protein (cereblon) is also required for their anti-myeloma and anti-inflammatory actions, including downregulation of TNF-alpha expression and T-cell immunomodulatory activity 4 . These studies strongly suggested that cereblon is not only a primary molecular target for the teratogenic effects of thalidomide-like drugs but also a target for anti-myeloma and immuno-modulatory activities of thalidomide and lenalidomide. Whether it will be possible to dissociate one effect from the other and what changes in thalidomide structure are required for achieving this goal remain obscure.

Supporters of lenalidomide approval for marketing in Brazil announced that a Cochrane Collaboration Centre Systematic Review (ordered by them) demonstrated that lenalidomide combined to dexamethasone is effective in multiple myeloma. Used in the context of a lobbying campaign to reverse the agency's decision this statement deviates the attention from the real prob- 
lem. The efficacy of lenalidomide in multiple myeloma was not questioned by ANVISA. A major reason why the Advisory Committee on Medicines (CATEME) recommended the agency not to approve the application was the lack of comparative clinical efficacy and safety trials demonstrating that lenalidomide is better than thalidomide.

Several randomized clinical trials showed that thalidomide and lenalidomide are effective, both as single agents and in combination with other drugs in multiple myeloma and other conditions. No randomized clinical trial however has compared efficacy and safety of lenalidomide versus thalidomide. A study by Gay et al. 5 (retrospective analysis of patients' medical records) compared the efficacy and adverse effects of lenalidomide plus dexamethasone versus thalidomide plus dexamethasone as the initial therapy for multiple myeloma and found that the former was somewhat more effective than the latter. Although being notoriously weak to support any general conclusion on the clinical superiority of one drug over the other, Gay et al.'s 5 study has been cited to support claims that lenalidomide is more effective and/or causes less peripheral neuropathy and deep vein thrombosis side effects than its prototype drug. Allegations of clinical superiority, however, need to be supported by data from gold standard comparative clinical trials of lenalidomide versus thalidomide (optimized dosage schedules) in therapeutic regimens for multiple myeloma and myelodisplastic syndrome.

Cost-effectiveness analyses (CEA) compare relative costs and outcomes of different medicines and therapies. Industry-sponsored CEA studies should be taken with caution for regulation purposes because their results can be biased by pharmaceutical companies. At any rate, there is a huge difference between Brazilian thalidomide (FUNED-MG) production costs and Celgene's lenalidomide marketing prices. Celgene's thalidomide (Thalidomid) is also much more expensive than the Brazilian drug. A study by Garrison et al. 6 addressed the problem of costeffectiveness of therapeutic regimens and found that life-time costs of regimens based on Revlimid were higher than those based on Celgene thalidomide. Costs of multiple myeloma treatment with lenalidomide have been estimated to exceed US\$160,000 per patient year.

Since clinical superiority of lenalidomide over thalidomide in multiple myeloma and other conditions remains undemonstrated, this analogue is - until proven to the contrary - a "metoo" of thalidomide. To approve sales in Brazil of a teratogenic thalidomide-like drug that offers no additional benefit in terms of efficacy and safety is unacceptable.

\section{Conflict of interests}

The author has received no honorarium, reimbursement for lectures, research grants or financial support of any kind from pharmaceutical companies.

1. Paumgartten FJ. Novel thalidomide analogues, "me too" drugs and the Brazilian law. Visa em Debate 2013; 1:2-10.

2. Helfand C. Top 20 orphan drugs by 2018. http:// www.fiercepharma.com/special-reports/top20-orphan-drugs-2018 (accessed on 23/Jul/2013).

3. Ito T, Ando H, Suzuki T, Ogura T, Hotta K, Imamura $\mathrm{Y}$, et al. Identification of a primary target of thalidomide teratogenicity. Science 2010; 327:1345-50.

4. Zhu YX, Kortuem KM, Stewart AK. Molecular mechanism of action of immune-modulatory drugs thalidomide, lenalidomide and pomalidomide in multiple myeloma. Leuk Lymphoma 2013; 54:683-7.

5. Gay F, Hayman SR, Lacy MQ, Buadi F, Gertz MA, Kumar S, et al. Lenalidomide plus dexamethasone versus thalidomide plus dexamethasone in newly diagnosed multiple myeloma: a comparative analysis of 411 patients. Blood 2010; 115:1343-50.

6. Garrison Jr. LP, Wang ST, Huang H, Ba-Mancini A, Shi H, Chen K, et al. The cost-effectiveness of initial treatment of multiple myeloma in the U.S. with bortezomib plus melphalan and prednisone versus thalidomide plus melphalan and prednisone or lenalidomide plus melphalan and prednisone with continuous lenalidomide maintenance treatment. Oncologist 2013; 18:27-36.

Submitted on $18 / \mathrm{Jan} / 2014$

Approved on 22/Jan/2014 